1 This preprint has not undergone peer review (when applicable) or any post-

2 submission improvements or corrections. The Version of Record of this article

3 is published in Nature Human Behaviour, and is available online at

4 https://doi.org/10.1038/s41562-022-01360-4

5

6 A view-only version of the final article is available here: $\underline{h t t p s: / / r d c u . b e / c O C b F}$

7

8

9

\section{Human value learning and representation reflects rational adaptation to task demands}

$$
\text { Keno Juechems }{ }^{1,2} \text {, Tugba Altun }{ }^{3} \text {, Rita Hira }{ }^{3} \text {, Andreas Jarvstad }{ }^{3}
$$

${ }^{1}$ Department of Experimental Psychology, University of Oxford, UK

${ }^{2}$ St John's College, University of Oxford, UK

${ }^{3}$ Department of Psychology, City University of London, UK

5296 words

4 figures.

Corresponding author: Andreas Jarvstad, andreas.jarvstad@city.ac.uk, College Building, City, University of London, Northampton Square, London EC1V 0HB, United Kingdom. 
27 When making decisions about goods and actions, humans and animals often rely on internally

28 represented values. However, to be useful across a wide range of contexts, these values need to be

29 represented on an absolute scale - a coding scheme that is computationally costly. By contrast,

30 representing values in a way that depends entirely on context is highly computationally efficient, but

31 can lead to irrational behaviour when values need to be compared across contexts. Thus, an efficient

32 learner would allocate limited computational resources only when needed according to their

33 expectations about the future. Here, we test the hypothesis that value representation is not fixed, but

34 rationally adapted to expectations in two human learning experiments. Unlike most lab-based tasks,

35 participants could use their initial experience (Phase 1) to optimise behaviour (Phase 2). Phase 1 was

36 designed to cause one group to expect only decisions within local contexts (relative code sufficient),

37 and another group to expect choices across local contexts (relative code insufficient). Despite

38 identical learning experiences, the group whose expectations included choices across local contexts,

39 went on to learn absolute value representations, and learned more absolute-like representations than

40 the other group. Human value representation is neither relative nor absolute, but efficiently and

41 rationally tuned to task demands.

42

43

44

45

46

47

48

49 
54 Across behaviour - from choosing what to eat ${ }^{1}$, to deciding whom to marry ${ }^{2}$, to choosing between stocks and bonds - value informs choice. In general, humans and other animals are adept at choosing between items across a wide range of item values. Historically, this ability has been conceptualized as being supported by a well-ordered absolute code. units of food, and a person might learn that one asset is worth $£ 100$ and another $£ 125$ (where the unit of measurement stays constant across time and contexts). A well-ordered and complete value code is optimal because it allows consistent selection of higher-valued actions: foraging in better patches, making better investments, and selecting better partners. As such, it is assumed in normative theories of decision-making ${ }^{3}$, optimal foraging theory ${ }^{4}$, computational models of learning ${ }^{5}$, and in key descriptive theories of choice ${ }^{6}$.

However, an absolute value code is computationally expensive, requiring encoding of a potentially infinite set of values: potentially reserving precious coding range for events that occur with low frequency. From the olfactory system in the fruitfly ${ }^{7}$, to visual systems ${ }^{8}$, to value coding in humans ${ }^{9}$ neural systems can overcome this problem by encoding input relative to the local context (and/or state ${ }^{10,11}$ ). The value of one foraging patch can, for example, be encoded relative to other nearby patches. Such context-dependent encoding is formalised in computational models, one broad and highly successful class of which is divisive normalisation ${ }^{12}$.

Computationally, divisive normalisation scales the current input by the local context and a constant, such that each input is scaled to the range of $(0,1)$ expressed as a fraction of maximum firing rate of a neuron (or pool of neurons). Our foraging animal could encode the value of Patch A as .25: $A_{\text {food }}=\frac{A}{1+A+B}$. This code is efficient, because it allows the animal to represent many different values with few resources (i.e., a small population of neurons ${ }^{12}$ ). Consider a new foraging context with very different patch values: Patch $Z=67$, Patch $W=200$. Although their absolute values are very different, these patch values can be encoded identically to the prior patch values. Z, for example, 
representation of a very large set of values with a small pool of resources in a context-dependent manner.

There is now ample evidence from psychology, behavioural ecology, primate neurophysiology and cognitive neuroscience that humans and other animals learn, and/or make choices consistent with such context-dependent value codes $\left({ }^{10,13-18}\right.$ but see $\left.{ }^{19}\right)$. A relative contextdependent code also describes the firing pattern of neurons in value-related areas of the prefrontal $\operatorname{cortex}^{20}$ and explains human errors of judgment across many domains ${ }^{17}$. Lately, these codes have also been shown to be efficient in the sense that they maximize mutual information under certain conditions $^{21}$.

However, although a relative code can be highly efficient, it can also lead to highly suboptimal decision-making. For example, having encoded the value of both Patch $\mathrm{A}$ and $\mathrm{Z}$ as .25, our foraging animal would be indifferent between Patch A (1 units of food) and Patch Z (67 units of food). Worse still, relative-value codes can lead to irrational preferences with animals preferring globally inferior options - as observed across species in many laboratory tasks ${ }^{10,15,17,22}$.

Given the potential for highly sub-optimal choice - how could such a flawed mechanism have evolved? It is possible that the ecology is such that opportunities for catastrophic consequences rarely arise. For many brain systems this seems plausible. For the perceptual system, for example, the temporal and spatial autocorrelation in brightness in natural scenes (e.g., day-night light cycle) mean that adapting to local brightness levels (e.g., dark adaptation ${ }^{23}$ ) is likely close to optimal.

For value encoding the benefits of a fixed relative code are less obvious. Values may be of course be context and/or state dependent ${ }^{24}$. For example, the value of water to an animal may depend on their level of thirst. However, unlike perceptual stimuli, values often need to be compared outside their immediate context. Examples include when an animal moves from one foraging context to another, or when people compare asset prices in one market to those in another market.

Here we attempt to reconcile optimal-but-costly absolute value codes, with efficient-butpotentially-irrational relative value codes. We propose that humans do not use a single fixed representation of value, but flexibly tune value codes based on their expectations what the codes are 
$107 f^{25}$. Further, we propose that the selection of which code to learn, is rational and efficient ${ }^{26}$. That is,

108 we propose that people learn relative codes when sufficient, and absolute-like codes when necessary.

109 Thus, we do not ask whether human value learning is absolute or relative overall $^{13,15}$, but

110 whether humans flexibly adapt $\mathrm{t}^{27,28}$ their value representation in a manner that can be explained by

111 expectation. In spirit, our work is similar to efforts in reinforcement learning to delineate under what

112 circumstances, and under what cost, humans switch from a habitual (model-free) representation to a

113 more costly representation that allows planning (model-based) ${ }^{29}$. To our knowledge, this question has

114 not been addressed in the field of value encoding despite its importance for optimal decision-making.

115 We tested the hypothesis that value representation rationally adapts to task demands in two

116 value-learning studies, in which human participants learned values of pair-wise presented items. We

117 implicitly manipulated task expectations, such that one group expected to make decisions within fixed

118 local contexts, and another group expected to make decisions across local contexts. A relative code is

119 sufficient for optimal decision-making in the former, but not the latter case. Despite identical learning

120 experiences, learnt value codes differed: participants learned more complex (absolute) representations

121 only when they expected it to be necessary, thus highlighting the rational and dynamic nature of value

122 representation.

Task and design

Participants took on the role of consultants to manufacturers of reproduction items (replicas of historical items). There were two separate manufacturers (of cars \& antiques) in two separate Phases

126 (Fig 1A). Participants' goal was to learn market prices in order to consult on which items to

127 manufacture. Participants learned item values through trial-by-trial feedback (Learning $1 \& 2$ ), after

128 which they advised the manufacturer in separate decision stages - without feedback (Decision $1 \& 2)$.

129 At the end, there were two surprise tasks (All-possible pairs, Value judgment) designed to measure

130 value encoding in Learning 2 (Phase 2).

131 There were two conditions. Participants were randomly and blindly assigned to either the Un-

132 crossed or Crossed group (green and blue respectively, Fig 1A). Each Phase begun with a Learning

133 stage, in which participants sampled market values (Fig 1B). A single mouse-click on an item

134 returned a single sale price (superimposed on the clicked item, Fig 1B). Participants were free to 
135 sample in any order and as much as they wished. Sampling for each pair was terminated by a

136 preliminary selling decision (Fig 1B), after which the next pair was shown. In each Phase, participants

137 learned the values of 6 items arranged into 3 pairs with normally distributed market prices (see Fig 1A

138 for details).

139 In the Decision Phases (Fig 1C), the Un-crossed group (green) made decisions about the pairs

140 they had previously experienced - without feedback. The Crossed group (blue) additionally made

141 decisions about novel pairs. These novel pairs were created by combining items previously

142 encountered in different local contexts during learning. Participants might, for example, decide

143 between Item $^{2}$ and Item $^{4}$ which had previously formed part of the first and second pair respectively.

144 Participants' choices in the Decision phases and surprise tasks were consequential (i.e., affected

145 payment, see Methods). 
A)

Phase 1 (antiques/cars)

Phase 2 (cars/antiques)

Phase 2 surprise tasks

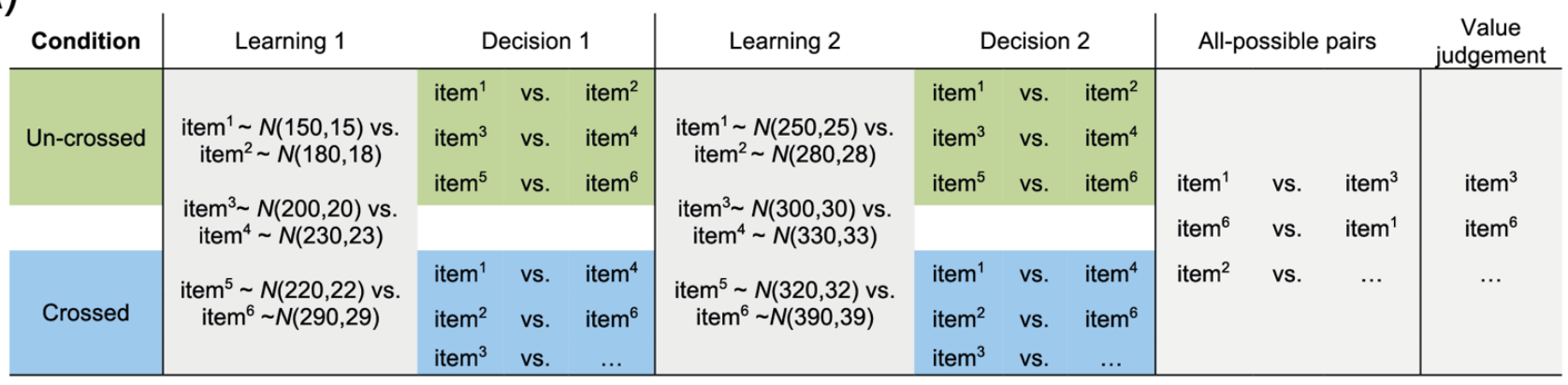

B)

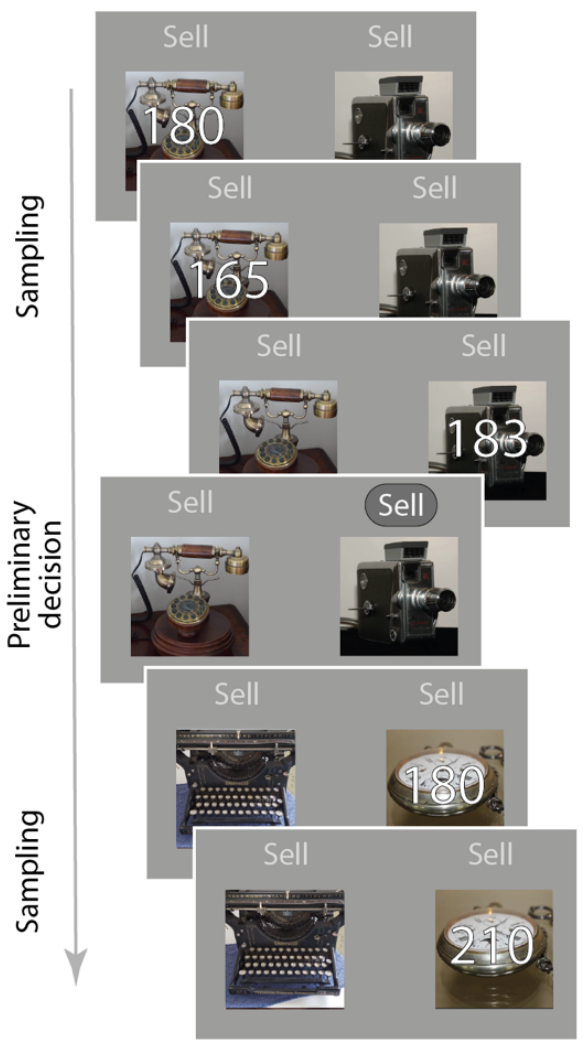

C)

Decision 1
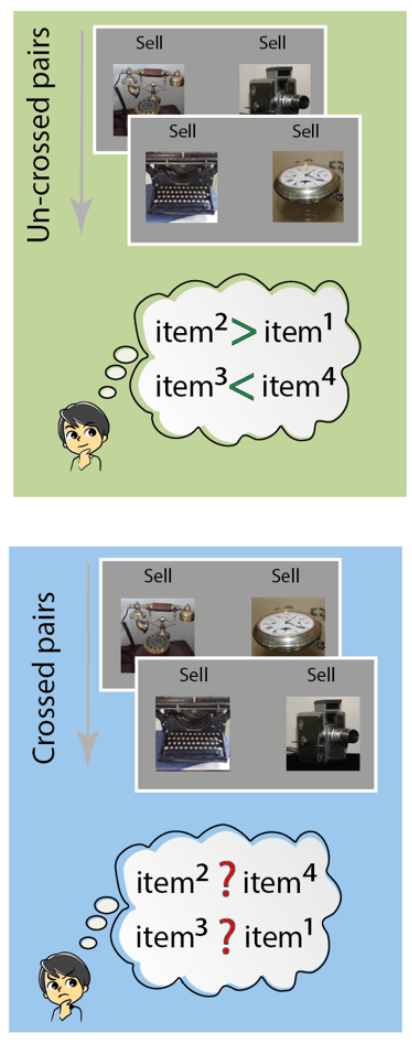

Learning 2
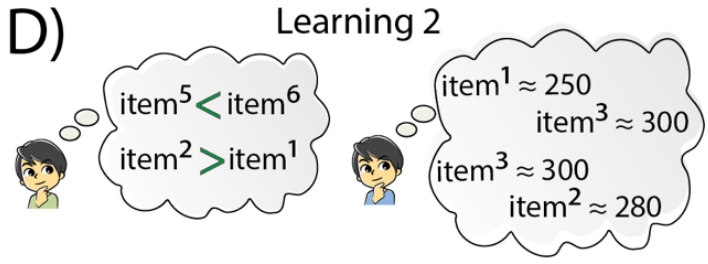

Decision 2

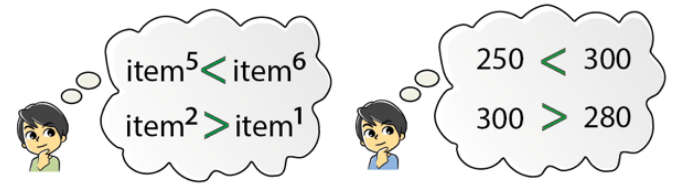

All-possible pairs

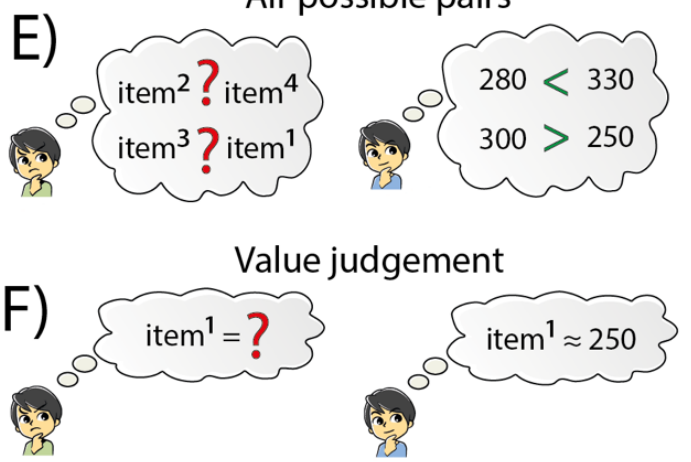

147 Figure 1 Experiment 1A (A) Each participant was assigned (double-blind) to either the Un-crossed (green

148 shade) or the Crossed (blue shade) condition. There were two Phases, which were structurally identical, but with

149 different market values and item types (counterbalanced). The mapping between item types and context was

150 randomized across participants, as was the item-value mapping. In each Phase, participants first learnt market

151 values of 6 items (antiques or vintage cars) arranged into 3 pairs. Participants learnt by sampling (B). A click on

152 an item returned a single sample. Participants were free to sample as much as they wished. Sampling for a given

153 pair ended once a preliminary selling decision was made. There were three sampling phases for each item-pair

154 (three preliminary decision/item). Item values were normally distributed (see A for details) with sampling

155 truncated at +- 2SD. The Learning phase was followed by decision-making (C), in which participants made

156 consequential decisions without feedback. The Un-crossed groups made decisions about previously sampled

157 item-pairs. The Crossed group also made decisions between novel item-pairings, composed of items from

158 different item-pairs. (D) We predicted that following the first decision phase, value-learning mechanisms would 
diverge in Phase 2 (D): the Un-crossed group would learn relative-value representations, whereas the Crossed

160 group would learn absolute-value representations. This divergence would cause the crossed group to make

161 better decisions and judgments in the final tasks (E-F). In All-possible pairs (E) participants made decisions

162 between all possible pairs from Phase $2(\mathrm{~N}=15$, repeated thrice for $\mathrm{N}=45$ ). In Value judgment (F), participants

163 judged the value the value of the six stimuli in Phase 2 presented in a random order by adjusting a slider

$164(\min =100, \max =450$, in integer steps) until it matched the perceived item value.

Recall that we hypothesized that people do not use a fixed value-learning mechanism, but

flexibly adapt their value-learning mechanisms to learn useful value-representations. As participants were blindly and randomly assigned to groups, both groups start Learning 1 with the same expectations. However, the first Decision phase, Decision 1 (Fig 1C), provides very different implicit signals for the two groups.

To see why, consider an extreme context-dependent encoding: a local ordering of values. Using this mechanism, one learns, for each pair, that one item is 'good' and that one item is 'bad'. That is, one learns the following (separate) sets of orderings: $\left[\right.$ Item $^{1}<$ Item $\left.^{2}\right],\left[\right.$ Item $^{3}<$ Item $\left.^{4}\right]$, and $\left[\right.$ Item $^{5}<$ Item $\left.^{6}\right]$. This learning mechanism will work well for the Un-crossed group (Fig 1C). It also works well in many published learning and decision-making tasks. This mechanism, however, will not work for participants in the Crossed group (Fig 1C, blue panel). Deciding between Item $^{2}$ and Item $^{4}$, for example, will be difficult as both would have been encoded as 'good'. different expectations, Decision 1 should therefore prompt the Un-crossed group to learn relativevalue representations (in Phase 2) and should cause the Crossed group to realise the insufficiency of this learning and cause them to adopt a more complex learning mechanism. Thus, the first Decision phase (Decision 1, Fig 1C) should trigger a shift in learning mechanisms in the second Phase, which would give rise to different value codes - measurable with the two final surprise tasks (Phase 2

184 Surprise tasks, Fig 1D,A). outperform Un-crossed in the penultimate All pairs task (Fig 1E), because an absolute-like encoding 
encoding of value should also cause Un-crossed to choose more irrationally when confronted with

189 specific diagnostic pairs (see below, and e.g., ${ }^{15}$ ).

190 In the Value judgement task, all Phase 2 items were presented sequentially, and participants

191 indicated their perceived value by adjusting a slider. This task was used to directly test the nature of

192 encoding, by contrasting the groups' value representations to those predicted by relative and absolute

193 encoding respectively, through representational similarity analysis (RSA) ${ }^{30,31}$.

\section{Decision-making performance}

As can be seen in Fig 2A, the Crossed group made better decisions in the surprise All pairs task: choosing the higher-value item in each pair more frequently than the Un-crossed group $(t(44)=$ $2.61, p=.012, C I=.026-.199, D=.77$, unpaired t-test). Thus, the Crossed group learned a value representation that allowed them to make better decisions in novel contexts (i.e., novel item-pairings). The difference in performance across the two groups shows that people flexibly adapt their valuelearning mechanism to expected task demands. result in diagnostic choice patterns for specific item-pairings. Specifically, in Phase 2, Item ${ }^{2}$ $\sim N(280,28)$ was paired with item ${ }^{1} \sim N(250,25)$. A relative learner would therefore learn that Item $^{2}$ is relatively 'good'. On the other hand, item ${ }^{3} \sim N(300,30)$ and item ${ }^{5} \sim N(320,32)$ were paired with highervalue items and were thus relatively 'bad'. A relative-value learner would prefer the locally 'good' (but globally inferior) Item ${ }^{2}$, to the globally superior (but locally 'bad') Item ${ }^{3,4}$ - thus exhibiting irrational choice ${ }^{\text {(see also e.g., 15). }}$ 
209

210 Figure 2. All-pairs task. A) Proportion high-value choices as a function of condition. Grey discs represent
A)

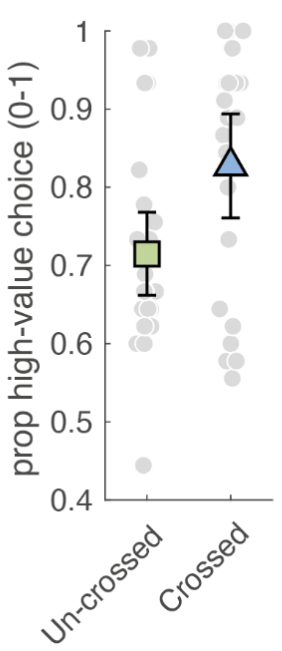

B)

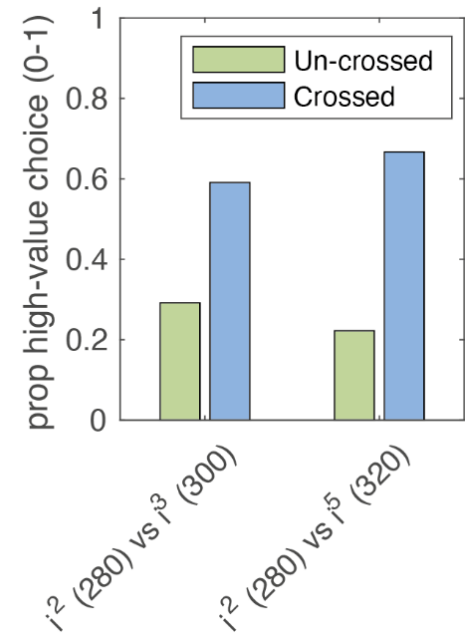

individual participants. Coloured symbols represent group means (green square $=$ Un-crossed, blue triangle $=$

212 Crossed) represent group averages. Error bars are 95\% CIs. B) Mean proportion high-value choices for a sub213 selection of highly diagnostic pairs, in which a local high-value item (item ${ }^{2}$ ) is globally inferior to other local 214 low-value items $\left(\right.$ item $^{3}$, item $\left.{ }^{5}\right)$.

The divergence in preferences for highly diagnostic pairs (Fig 2B) match those predicted from

217 relative and absolute value encoding respectively. The Un-crossed group prefer the globally inferior option, choosing it instead of the globally superior options (preferring Item $^{2}$ to Item $^{3}$, and to Item ${ }^{5}$ ), whereas the Crossed group express a weak preference for the globally superior items. The difference between groups was marginal for the first pair $(U=183, p=.055, r=.307$, two-tailed Mann-Whitney $\mathrm{U})$, and highly significant for the second pair $(U=145, p=.003, r=.451$, two-tailed Mann-Whitney U), with medium effect sizes.

Thus, we replicated the tendency for people to learn globally irrational value codes ${ }^{\text {e.g., }} 15$ in the 'Un-crossed' group. However, when people can form expectations that the task may involve choices across local contexts, they learn more globally optimal codes - thus showing rational adaptations to expected task demands.

\section{Value representation}

The previous analyses suggest that human choice is consistent with 1) value encoding being dependent on expected task demands, and 2) that the selection of which codes to learn is rational and 
efficient. Representational similarity analysis (RSA) ${ }^{30,31}$ applied to the final judgement task (Fig 1A),

231 allowed us to more directly address the question of value representation.

We computed representation dissimilarity matrices (RDMs) separately for each participant, which were then averaged to group-wise RDMs (Fig 3A, Methods). These value RDMs, show each group's value representation in the form of a dissimilarity structure. The structure shows the dissimilarity of all possible pairs of items. The items are ordered not by their item number but by their objective value. A dissimilarity of 0 implies that item values are represented identically (item pairs along the diagonal), and a dissimilarity of 1 implies that item values are maximally dissimilar. Empirical RDMs are most readily interpreted when compared to model RDMs derived from a priori assumptions. For this purpose, we defined two model RDMs and compare their predictions (using rank-correlations) to the RDMs derived from participants' behaviour, as is standard practice ${ }^{31}$. value of the left item is item value left $^{\text {le }}=\frac{\text { item }^{\text {left }}}{1+\text { item }^{\text {left }}+\text { item }^{\text {right }}}$. Thus, the value of the left item depends on the value of the right item (and vice versa). Variants of this equation are used across disciplines to formalize context-dependent encoding ${ }^{12}$.

The second model, the Absolute-value RDM (Fig 3A), formalizes absolute value encoding. The model assumes that participants learn the expected values of items. For example, the Absolute model

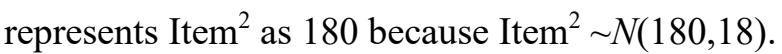

As can be seen (Fig 3A), the Relative and the Absolute model RDMs predict different dissimilarity structures. On the one hand, the structure of the Absolute RDM simply reflects the objective values: items with larger value differences are encoded as more dissimilar. On the other hand, the Relative RDM has clusters of items that are objectively similar in value but are nonetheless encoded as highly dissimilar. The Relative RDM also captures the predicted (and observed, Fig 2B) 'irrational' value encoding by which Item $^{2}$, for example, is encoded as more dissimilar to Item $^{3}$ than 254 to Item $^{4}$.

The RDM for the Un-crossed group' (Fig 3A) shares many features with the Relative model. 
the correlations between each model and the two groups allows for a more formal assessment

258 (Methods). A large positive correlation between a specific participant's RDM and model RDM, shows

259 that their representation of value is well accounted for by the model in question (and conversely a low

260 correlation shows that the model accounts poorly for their representation).

A)

A) Un-crossed RDM
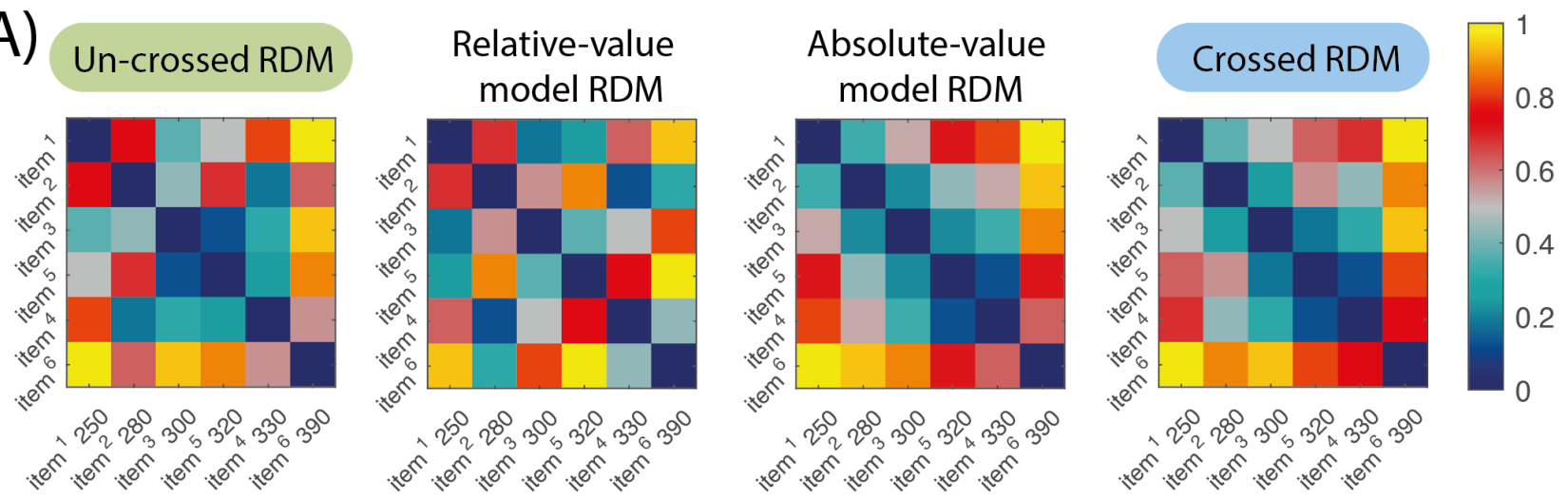

खen

ver
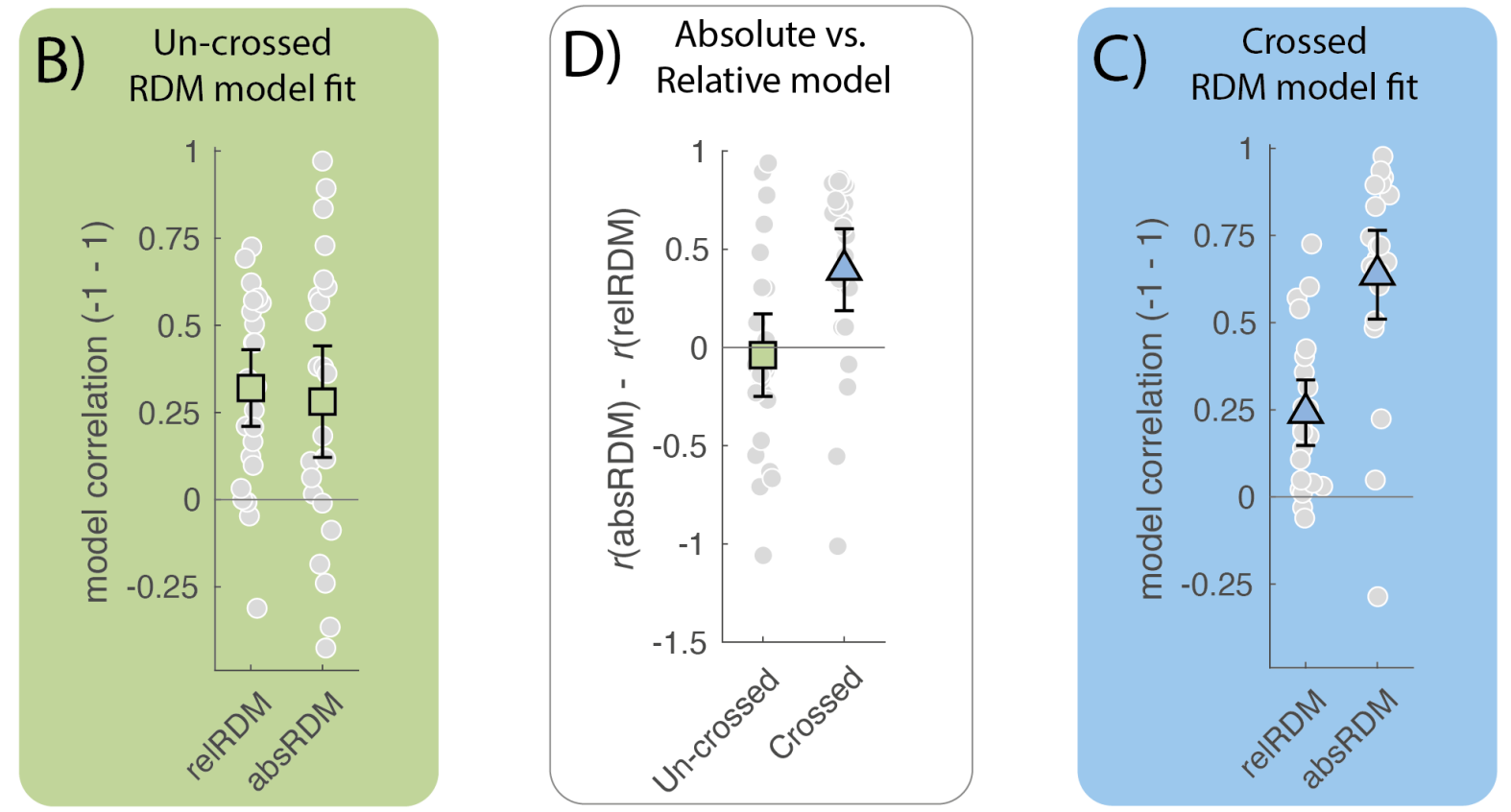

Figure 3. Value representation. A) Rank-transformed and scaled empirical and model RDMs (Methods): Uncrossed group RDM (green), Relative-value model RDM, Absolute-value model RDM, Crossed group RDM (blue). The colour-bar indicates dissimilarity $(0=$ minimal dissimilarity, $1=$ maximal dissimilarity $)$. B-C) Model correlations: Individuals are plotted as grey discs, with jittered $\mathrm{x}$-axes for improved legibility. Coloured symbols (box=green, triangle=blue) indicate group means. Error bars are 95\% CIs. B) Un-crossed group model correlations. C) Crossed group model correlations. D) Absolute vs Relative model correlations. Positive values indicate larger absolute-value model correlations. Negative values indicate a better relative-value model fit. BC) 
Fig 3B shows group-averages (green squares) and individuals' (grey discs) model correlations

272 for the Un-crossed group. Fig 3C shows the same for the Crossed group (blue triangles=averages,

273 grey discs=individuals). There appears to be a slight advantage for the relative model in the Un-

274 crossed group (Fig 3B) and a marked advantage for the absolute model in the crossed group (Fig 3C).

275 To select among the two models, one should consider the relative advantage of one model over the other. This is plotted in Fig 3D. Positive differences indicate that the absolute model better accounts for participants' representation. Conversely, negative differences indicate that the relative model is 278 better.

Although many in the Un-crossed group are best accounted for by relative-value representation, a minority (10 of 24) in this condition are better described as absolute-value learners (negative and positive $r$ 's respectively, Fig 3D). This results in a non-significant trend towards relative-value representations $(t(23)=-.36, p=.729, C I=-.260-.183, D=.074$, one sample t-test against 0$)$. For the Crossed condition there is a marked and highly significant tendency to learn absolute-value representations $(t(21)=3.73, p=.0012, C I=.175-.617, D=.794$, one-sample t-test against 0$)$, with a large effect size.

When contrasting the two groups (Fig 3D), it is clear that Crossed group learned more absolutelike representations than the Un-crossed group $(t(44)=2.88, p=.0062, C I=.130-.739, D=.849$, unpaired t-test). Jointly, the results show that people adapt their learning to expected task demands, and that people only learn absolute-like value representations when a relative representation is insufficient for the task at hand.

\section{Choice and value representation in a binomial task}

Although values often come from continuous distributions as they did in Experiment 1 (e.g., market prices, food quantities, attractiveness of potential partners), laboratory tasks often involve binomial outcome distributions, often with a fixed number of trials ${ }^{15,32,33}$. Next, we therefore sought to establish whether people can flexibly tune their value-learning mechanism also in this case. experiences were identical across conditions, Phase 1 set participants expectations for Phase 2 in a 
surprise tasks (All possible pairs, Value judgement), with the notable exception that value

300 distributions were binomial, the number of 'samples' from each distribution was fixed across

301 participants, and the experiment was run online (see Methods for details).

302 Based on Experiment 1, we predicted that - relative to the Un-crossed group - the Crossed

303 group would show better All-possible pairs task performance, improved choice for the single

304 diagnostic item pair in this experiment, and learn more absolute-like value representations as

305 identified through RSA.

306

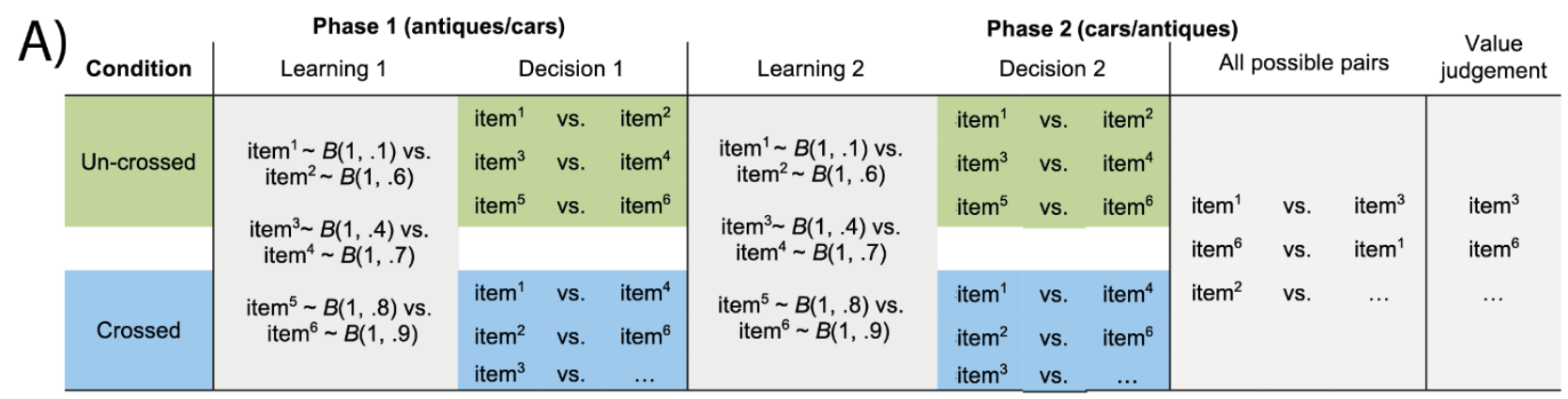

B)

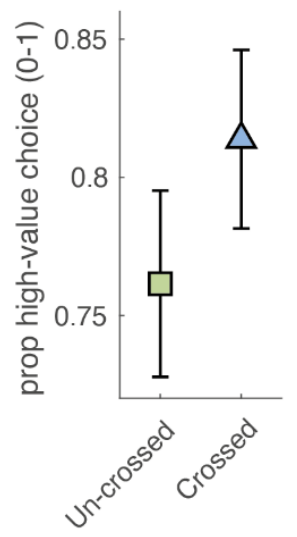

$30^{\circ}$

\section{C)}

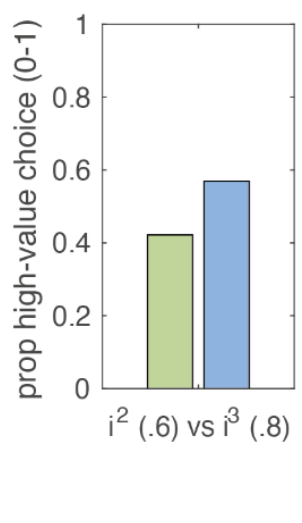

D) Un-crossed RDM

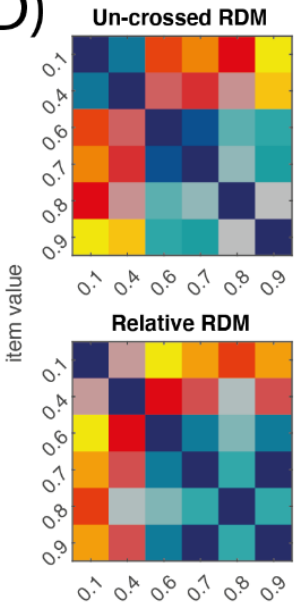

0.51

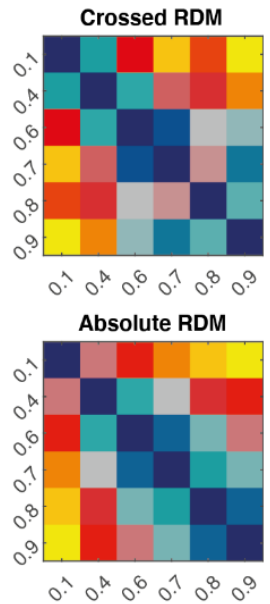

item value
E)

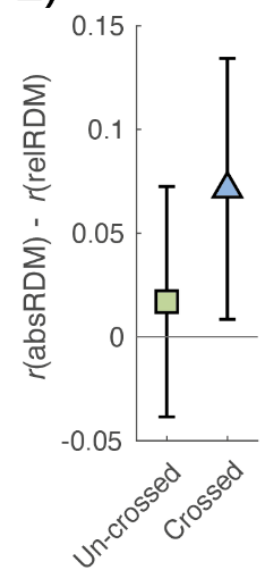

Figure 4. A) Key design features were identical to Experiment 1. Each participant was assigned (double-blind)

309 to either the Un-crossed (green shade) or the Crossed (blue shade) conditions. There were two Phases, which

310 were structurally identical, but with different market values and item types. In each phase, participants first

311 learnt market values of 6 items (antiques or vintage cars) arranged into 3 pairs. The Learning phase was

312 followed by decision-making, in which participants made consequential decisions without feedback. The Un-

313 crossed groups made decisions about previously sampled item-pairs. The Crossed group made decisions

314 between novel item-pairings, composed of items from different previously sampled item-pairs. B) All possible

315 pairs 2AFC proportion high-value choices as a function of condition. Coloured symbols represent group means

316 (green square $=$ Un-crossed, blue triangle $=$ Crossed) represent group averages. Error bars are $95 \%$ CIs. C) 
317 Mean proportion high-value choices for a sub-selection of highly diagnostic pairs, in which a local high-value

318 item $\left(\right.$ item $^{2}$ ) is globally inferior to a local low-value items (item ${ }^{3}$ ) or a local-high value item (item ${ }^{5}$ ). D) Rank-

319 transformed and scaled empirical and model RDMs (Methods). The colour-bar indicates dissimilarity

320 ( 0 =minimal dissimilarity, $1=$ maximal dissimilarity). D) Absolute vs Relative model correlations. Positive values

321 indicate larger absolute-value model correlations. Negative values indicate a better relative-value model

322 correlation. Error bars are 95\% CIs.

323

As in Experiment 1, the Crossed group made significantly better decisions when choosing between all possible pairs (Fig. 4B, $t(120)=2.19, p=.015, C I=.005-.099, \mathrm{D}=.40$, one-tailed unpaired t-test). Thus, the Crossed group learned a higher-fidelity value representation - despite having the exact same learning experience. The improved performance for diagnostic item-pairs for the Crossed group also replicated. In Experiment 2, a single pair was highly diagnostic (Item ${ }^{2} \sim B(1$, .6) \& Item $\left.{ }^{4} \sim B(1, .8)\right)$. A relative learner would encode the items in this pair as 'good' and 'bad' respectively, because in their local pairing the lower-probability Item $^{2}$ was relatively good, and the higher-probability Item $^{4}$ was relatively bad. As in the first experiment, participants in Crossed chose the better item more frequently than those in Un-crossed (Fig. 4C; $U=1538, p=.038, r=.17$, onetailed Mann-U Whitney test).

Fig 4D shows the group RDMs, alongside Relative and Absolute model RDMs. As can be seen, the Crossed group's dissimilarity structure is highly similar to the Absolute RDM. The Uncrossed groups' dissimilarity structure shares many features with the Relative RDM, but also shares some features with the Absolute RDM. For example, in the Un-crossed group item values $B(1, .8)$ and $B(1, .4)$ are represented as similar (as predicted by Relative model, Fig 4D), but item values $B(1, .9)$ and $B(1, .1)$ are represented as most dissimilar among all pairs (as predicted the Absolute Model, Fig 4D).

This overall pattern is confirmed by a formal comparison (Fig 4D): the Un-crossed group is

342 not consistently better captured by either model $(t(63)=.601, p=.275, C I=-.039-.074, D=.08$, one343 tailed). However, the crossed group is better accounted for by the absolute model $(t(57)=2.23, p=$ $344.015, C I=.007-.136, D=.29$, one-tailed), and marginally better accounted for by it than the Uncrossed group $(t(120)=-1.27, p=.103, C I=-.139-.030, D=.23$, one-tailed $)$. Thus, the key findings 
346 in the main study replicated in an online study, without free sampling and with binomial value

347 distributions.

\section{Discussion}

We sought to reconcile the theoretical and empirical tension between two diametrically opposing accounts of value learning and encoding: a rational but computationally costly absolute value representation $^{3,4,6}$, and an efficient, but potentially irrational, relative value representation $9,12,13,15$. We proposed that humans (and possibly other animals) do not use a single fixed mechanism learning either absolute or relative value codes - but adapt their learning to expected task demands in an efficient and rational manner: learning sufficient and necessary value representations.

We tested this hypothesis in two human value-learning experiments: one involving normally distributed values and the other binomial values. In each study, the first Phase was equivalent to the full experience of participants in many experimental paradigms ${ }^{(\mathrm{eg.g} .15,32)}$. The second Phase gave participants the chance to use their prior experience with the task to tune their learning mechanism to optimise task performance. Phase 2 thus mimicked the opportunity to adapt and tune learning mechanisms that arise in many real-life tasks (that are performed more than once).

Despite identical learning experiences, the two groups learned different value codes.

362 Specifically, across the two studies, the Crossed group made decisions that are consistent with a higher-fidelity representation (Fig 2A, 4B), made fewer irrational choices (Fig 2B,4C), and learned value representations that were more absolute-like than the Un-crossed group (Fig 3,4D-E). Importantly, participants learned more complex (absolute) representations only when they expected it to be useful. Thus, people do not learn either absolute or relative value codes but adapt their learning to what they expect to use the code for.

What are the limits of the flexibility demonstrated by our participants? For the Un-crossed condition, we saw that a minority of participants also learned absolute-like codes, and some in the Crossed condition persisted with relative encoding - even though it was clearly sub-optimal. Such individual differences may be driven by cognitive capacity limitations ${ }^{34}$, by intrinsic computational noise $\mathrm{e}^{35,36}$, by mechanisms relating to working memory or attention ${ }^{37,38}$, or the belief that item-specific value encoding was relevant (despite the absence of such instruction). Future work might manipulate 
task demands, or measure cognitive capacity, to address these questions. Additionally, given that task difficulty affect the nature of learnt value codes ${ }^{33 \text {, (c.f. } 39)}$, it is likely that overall task difficulty modulate people's ability to switch between value codes.

A second outstanding question is the learning mechanisms that give rise to the flexible and adaptive value representations we observe. Our studies were designed for extremely well-controlled measurement of value representation following learning. The trade-off is that the design is not effective in characterizing learning mechanisms themselves - as opposed to the codes they give rise to.

It is possible, that a single mechanism underlies the observed flexibility in value encoding.

383 Such a mechanism could be implemented with a free parameter governing the extent to which

384 learning is relative, for example by extending Equation 1, such that item ${ }^{\text {left }}=$ $\frac{\text { item }^{\text {left }}}{1+w *\left(\text { item }^{\text {left }}+\text { item }^{\text {right }}\right)}$, were $w$ is a free parameter between 0 (for wholly absolute encoding) and 1 (for wholly relative encoding). However, it is also possible that mechanisms rely at least in part on different cognitive substrates as in, for example, model-based and model-free learning ${ }^{40-42}$. Future work is needed to address the question of mechanism, and perhaps more importantly mechanism selection, which likely requires higher-level cognition and monitoring of goals. Extrapolating beyond value encoding and representation, our work highlights the potential for

391 key phenomena to reflect task-specific adaptations. To what extent do inferred mechanisms reflect

392 fundamental and fixed properties of the neural system under study ${ }^{\text {(see also } 43)}$ ? And/or to what extent do

393 inferred mechanisms reflect specific adaptations to the task at hand? The answer likely depends in

394 part on the degrees of freedom of the experimental task itself - the extent to which behaviour could

395 have been different. In some experimental paradigms those degrees of freedom are likely small (e.g.,

396 perceptual contrast discrimination), and in others they may be larger ${ }^{\text {(e.g., } 44)}$.

398 representation and resolve the tension between optimal-but-costly absolute value encoding, and

399 efficient-but-potentially-irrational, relative value encoding. Humans do not simply have a single, fixed

400 form of representation, but rather adjust their code in a resource-efficient ${ }^{45-47}$ manner according to 
expected task demands. Further, our findings highlight that both absolute and relative codes

402 previously found can be explained by the fact that participants infer which code would be sufficient

403 for the current task. Value encoding is neither absolute nor relative but depends on what values are 404 for

\section{Methods}

406

407

408

409

410

\section{Experiment 1 - Participants}

The study was approved by the local ethics committee at City, University of London. Sixty participants were recruited via the local participation panel. Participants provided written informed consent and were debriefed. Participants had normal, or corrected-to-normal, vision, were fluent in English, healthy (no known physical or psychological conditions), and between 18-40 years old. We excluded participants who did not fulfil minimal task requirements. Criteria apply to the Learning phases only (Fig 1A,B), and are therefore orthogonal to the target behaviour in the final tasks (Fig 1A). Exclusion criteria were based on 1) sampling behaviour and 2) below-chance performance for the preliminary decisions in the first sampling phase. Participants who only sampled once (or fewer), per item per item-pair sampling opportunity, were excluded (Learning 1-2, Fig 1).

This cut-off represents $<=18$ samples per Context and is far lower than the median of $123(I Q R=118)$ and $143(I Q R=91)$ for Phase 1 and 2 respectively. There were 9 preliminary decisions during Learning (3 pairs presented thrice, Fig 1A,B). Random behaviour would result in a proportion of high-value choice between .22 and .78 (95\% CI). Participants who performed more poorly than chance were excluded. In summary, we excluded participants who showed no or little evidence of learning - a precondition for encoding value (whether in an absolute or relative form).

In total, fourteen participants met one or both criteria for a final sample size of $n=46: 24$ of which were assigned to the 'Un-crossed' condition, and 22 of which were assigned to the 'Crossed' condition.

\section{Experiment 1 - Materials}

Participants took on the role of a consultant to a manufacturer of reproduction items in two different contexts (antiques/cars, Fig 1). The item-values and item-pairs were Phase-specific (Fig 1A). However, the mapping of item type (antiques/cars) to Phase, the mapping of specific items (e.g., 
typewriter) to item-values (e.g., $N(180,18))$, and the side on which items were presented during

430 sampling, were all randomized across participants.

Item-values (Fig 1A) were selected primarily so that absolute-value and relative-value representations dissociate (Fig 1-3, Main text), and secondarily to achieve a balance between taskdifficulties in the Learning and Decision phases. A single sample from one item resulted in a draw from the corresponding normal value distribution (truncated at \pm 2 SD). The Learning phases (Fig 1A,B,D) were self-paced, and participants had a wide range of different strategies as evidenced by the wide range of the number of samples drawn (range Phase 1: $\min =48, \max =478$ : range Phase 2; $\min =32, \max =509)$.

The Decision phases (Fig 1A,C,D) involved 18 decisions per Phase. The Un-crossed group decided between the pairs they had experienced during sampling (repeated 6 times). The Crossed group made decisions between novel pairs ( 6 novel pairs x $2=12$ decisions, see Fig 1 for examples), in addition to learnt pairs ( 3 pairs $\times 2=6$ decisions). The novel pairs for this group were drawn randomly from the possible novel combinations and thus varied from participant to participant. The two final tasks (Fig 1A,E) were identical across groups. The All-possible pairs task involved 15 pairs, representing a full factorial combination of all possible pairs from Phase 2 (excluding identical pairs), repeated thrice for a total of 45 pairs. The Value judgment task involved the 6 items in Phase 2, presented one at a time along with a slider-interface $(\min =100, \max =450)$. For all tasks, the presentation order and presentation side (where applicable) were randomized across participants.

\section{Experiment 1 Procedure}

Participants read the information sheet, provided written informed consent and completed the tasks. After completing the behavioural tasks, participants completed three questionnaires. These formed parts of one author's MSc dissertation project and are not analysed here.

\section{Experiment 1 Apparatus}

Stimuli were displayed on touchscreens (Ilyama T2245MSC) and code was written in MATLAB (Mathworks) using PsychToolbox ${ }^{48}$ on Linux (Xubuntu 18.04) with a soft real-time kernel. 

participants were recruited via Prolific. Participants were fully informed, provided written informed consent and were debriefed. Participants had normal, or corrected-to-normal, vision, were fluent in English, healthy (no known physical or psychological conditions), and between 18-40 years old. experienced the same number of trials, sampling behaviour cannot be used for excluding disengaged participants (unlike Experiment 1). Instead, we excluded participants who did not learn to choose among the pairs experienced during Learning. Note that both relative and absolute mechanisms can learn to choose between presented pairs, resulting in exclusion criteria orthogonal to learning mechanisms. We excluded participants that made more than one error when choosing between the Learning Phase item-pairs in the final All-possible Pairs task, as well as those who made identical probability-of-sale judgements for all stimuli in the Judgement task, for a final sample of $n=122$ (i.e., participants had to choose the higher-valued item five out of six times to be included, allowing for a single mistake but otherwise appropriate learning): of which 64 participants were 'Un-crossed' and 58 were 'Crossed'.

\section{Experiment 2 Materials and Procedure}

As in Experiment 1, participants took on the role of a consultant to a manufacturer of reproduction items in two different contexts (antiques/cars, Fig 4). Key design features were identical to Experiment 1. However, outcomes were binomial (successful sale/unsuccessful sale), the task was not self-paced, and the learning experience was not 'blocked' by item-pairs (item-pairs were randomly intermixed during learning) and involved a relatively rapid stimulus display sequence. In the Learning Phases, participants saw each item pair presented side-by-side $(\sim 1 \mathrm{sec})$, followed by a response phase in which participants had $\sim 1.5$ second to make a choice, followed by sequential feedback, in which the chosen item was presented first followed by the unchosen item.

481 Outcome feedback was in the form of a green image outline (successful sale) or a red image outline 482 (unsuccessful sale). In Learning 1 (Fig 4A), there were 3 pairs in a single block of 30 trials. In 483 Learning 2 (Fig 4A) there were 3 pairs in two blocks for a total of 60 trials. 
485 This was achieved by pre-allocating and shuffling an outcome vector (of 1's and 0's) for each item.

486 This design minimizes the impact of sampling error ${ }^{49}$ on differences between participants and/or 487 conditions.

488 The Decision phases involved 12 decisions without feedback and were run between each block of trials (once for Learning 1, and twice for Learning 2). The Un-crossed group made decisions

490 between pairs experienced during learning, and the Crossed group made decisions also between novel 491 pairs composed of items from different learning pairs.

492 The two final tasks (Fig 1A,E) were identical across groups. The All-possible pairs task 493 involved 15 pairs, representing a full factorial combination of all possible pairs from Phase 2 494 (excluding identical pairs), repeated twice for a total of 30 pairs. The Value judgment task involved the 6 items in Phase 2, presented one at a time along with a slider-interface $(\min =0 \%$, $\max =100 \%)$ representing the probability of an item selling. The presentation order and presentation side were

497 randomized across participants for all tasks.

Design and Statistical Analyses

Both experiments used a between-subject design with participants assigned randomly and

500 blindly to one of two conditions: Un-crossed and Crossed. Our analyses focus on differences between 501 the two groups for the two final tasks (Fig 1-3), and within-task contrasts against reference magnitudes. The primary inferential statistic was the t-test. T-tests are relatively robust and were used whenever feasible (Fig 2A, Fig 3D, Fig 4B,E). For data with clear deviations from parametric assumptions (Fig 2B), less powerful non-parametric tests were used. All reported tests for Experiment 1 are two-tailed. Predictions for Experiment 2 were derived from results from Experiment 1 and are therefore one-tailed. Reported effect sizes are Cohen's $D$ for t-tests and rank-biserial correlation $r$ for non-parametric tests, with standard interpretations. Standard RSA protocols ${ }^{31}$ were followed. Value RDMs were computed as the Euclidean distance between judged values. Group-average RDMs were computed by averaging (arithmetic

510 mean) over participants' RDMs. Model RDMs were computed as the Euclidean distance between 1) 511 item values defined by Equation 1 (Relative RDM), and 2) the expected values (Absolute RDM). 
513 scaled to $0-1$, where 0 implies identical item-values and 1 means maximally dissimilar item-values.

514 We computed the distance between model RDMs and participant RDMs (Fig 3B-D) by Kendall-Tau

515 rank correlation. Large positive $r$ 's imply a high degree of similarity between participants value

516 representation and model RDMs (and $r=0$ implies no relationship).

517 All statistical analyses were performed in MATLAB 2020b (MathWorks).

518 Data availability

519 Data is available online on OSF (LINK here upon publication, contact authors for code for 520 review).

521 Code availability

522 Code for all analyses is available on OSF (LINK here upon publication, contact authors for 523 code for review).

Author Contributions

$525 \mathrm{KJ}, \mathrm{AJ}$ designed the research; TA, RH conducted the research; AJ analysed the data; KJ, AJ

526 contributed materials/analysis tools and wrote the paper.

\section{Acknowledgements}

528 Andreas Jarvstad was supported by a British Academy Postdoctoral Fellowship in the development stage (D-MAD, PF150005). We thank Peter Barr for programming Experiment 2. 
531 1. Clithero, J. A. \& Rangel, A. Informatic parcellation of the network involved in the computation of 532 subjective value. Social Cognitive and Affective Neuroscience 9, 1289-1302 (2014).

533 2. Darwin, C. On marriage. https://www.darwinproject.ac.uk/tags/about-darwin/family-life/darwin534 marriage\# (1838).

535 3. Morgenstern, O. \& Von Neumann, J. Theory of games and economic behavior. (Princeton $536 \quad$ university press, 1953).

537 4. Stephens, D. W. \& Krebs, J. R. Foraging theory. vol. 1 (Princeton University Press, 1986).

538 5. Sutton, R. S., Barto, A. G., \& others. Introduction to reinforcement learning. vol. 135 (MIT press 539 Cambridge, 1998).

540 6. Tversky, A. \& Kahneman, D. Advances in prospect theory: Cumulative representation of uncertainty. Journal of Risk and uncertainty 5, 297-323 (1992).

542 7. Olsen, S. R., Bhandawat, V. \& Wilson, R. I. Divisive Normalization in Olfactory Population Codes. Neuron 66, 287-299 (2010).

544 8. Heeger, D. J. Normalization of cell responses in cat striate cortex. Vis Neurosci 9, 181-197 (1992).

545 9. Khaw, M. W., Glimcher, P. W. \& Louie, K. Normalized value coding explains dynamic adaptation 546 in the human valuation process. Proc Natl Acad Sci USA 114, 12696-12701 (2017).

547 10. Pompilio, L. State-Dependent Learned Valuation Drives Choice in an Invertebrate. Science 311, $548 \quad 1613-1615(2006)$.

549 11. Karni, E., Schmeidler, D. \& Vind, K. On State Dependent Preferences and Subjective $550 \quad$ Probabilities. Econometrica 51, 1021 (1983).

551 12. Carandini, M. \& Heeger, D. J. Normalization as a canonical neural computation. Nat Rev $552 \quad$ Neurosci 13, 51-62 (2012).

553 13. Stewart, N., Chater, N. \& Brown, G. D. A. Decision by sampling. Cognitive Psychology 53, 1-26 554 (2006).

555 14. Yamada, H., Louie, K., Tymula, A. \& Glimcher, P. W. Free choice shapes normalized value 556 signals in medial orbitofrontal cortex. Nat Commun 9, 162 (2018). 
15. Klein, T. A., Ullsperger, M. \& Jocham, G. Learning relative values in the striatum induces violations of normative decision making. Nat Commun 8, 16033 (2017).

16. Palminteri, S. \& Lebreton, M. Context-dependent outcome encoding in human reinforcement learning. https://osf.io/4qh2d (2021) doi:10.31234/osf.io/4qh2d.

17. Rigoli, F. Reference effects on decision-making elicited by previous rewards. Cognition 192, 104034 (2019).

18. Rigoli, F., Mathys, C., Friston, K. J. \& Dolan, R. J. A unifying Bayesian account of contextual effects in value-based choice. PLoS Comput Biol 13, e1005769 (2017).

19. Gluth, S., Kern, N., Kortmann, M. \& Vitali, C. L. Value-based attention but not divisive normalization influences decisions with multiple alternatives. Nature Human Behaviour 4, 634645 (2020).

20. Rustichini, A., Conen, K. E., Cai, X. \& Padoa-Schioppa, C. Optimal coding and neuronal adaptation in economic decisions. Nat Commun 8, 1208 (2017).

21. Bhui, R. \& Gershman, S. J. Decision by sampling implements efficient coding of psychoeconomic functions. Psychological Review 125, 985 (2018).

22. Bavard, S., Rustichini, A. \& Palminteri, S. Two sides of the same coin: Beneficial and detrimental consequences of range adaptation in human reinforcement learning. Sci. Adv. 7, eabe0340 (2021).

23. Normann, R. A. \& Werblin, F. S. Control of retinal sensitivity: I. Light and dark adaptation of vertebrate rods and cones. The Journal of general physiology 63, 37-61 (1974).

24. McNamara, J. M., Trimmer, P. C. \& Houston, A. I. The ecological rationality of state-dependent valuation. Psychological Review 119, 114-119 (2012).

25. James, W. The principles of psychology. vol. 1 (Henry Holt \& Co, 1890).

26. Anderson, J. R. The adaptive character of thought. (Psychology Press, 2013).

27. Payne, J. W., Bettman, J. R. \& Johnson, E. J. The adaptive decision maker. (Cambridge university press, 1993).

28. Anderson, J. (1990). The adaptive character of thought. Hillsdale, NJ: Erl-baum. 
584

585

586

587

588

589

590

591

592

593

594

595

596

597

598

599

600

601

602

603

604

605

606

607

608

609

610

611

29. Kool, W., Gershman, S. J. \& Cushman, F. A. Cost-Benefit Arbitration Between Multiple Reinforcement-Learning Systems. Psychol Sci 28, 1321-1333 (2017).

30. Kriegeskorte, N., Goebel, R. \& Bandettini, P. Information-based functional brain mapping. Proceedings of the National Academy of Sciences 103, 3863-3868 (2006).

31. Nili, H. et al. A Toolbox for Representational Similarity Analysis. PLoS Comput Biol 10, e1003553 (2014)

32. Hertwig, R., Barron, G., Weber, E. U. \& Erev, I. Decisions From Experience and the Effect of Rare Events in Risky Choice. Psychological Science 15, 6 (2004).

33. Bavard, S., Rustichini, A. \& Palminteri, S. The construction and deconstruction of sub-optimal preferences through range-adapting reinforcement learning.

34. Shenhav, A. et al. Toward a rational and mechanistic account of mental effort. Annual review of neuroscience 40, 99-124 (2017).

35. Prat-Carrabin, A. \& Woodford, M. Efficient coding of numbers explains decision bias and noise. http://biorxiv.org/lookup/doi/10.1101/2020.02.18.942938 (2020) doi:10.1101/2020.02.18.942938.

36. Juechems, K., Balaguer, J., Spitzer, B. \& Summerfield, C. Optimal utility and probability functions for agents with finite computational precision. Proceedings of the National Academy of Sciences 118, (2021).

37. Spektor, M. S., Gluth, S., Fontanesi, L. \& Rieskamp, J. How similarity between choice options affects decisions from experience: The accentuation-of-differences model. Psychological Review 126, 52-88 (2019).

38. Collins, A. G. E. \& Frank, M. J. How much of reinforcement learning is working memory, not reinforcement learning? A behavioral, computational, and neurogenetic analysis: Working memory in reinforcement learning. European Journal of Neuroscience 35, 1024-1035 (2012).

39. Edwards, D. J., Pothos, E. M. \& Perlman, A. Relational versus absolute representation in categorization. Am J Psychol 125, 481-497 (2012).

40. Collins, A. G. E. \& Cockburn, J. Beyond dichotomies in reinforcement learning. Nat Rev Neurosci 21, 576-586 (2020). 
612 41. Russek, E. M., Momennejad, I., Botvinick, M. M., Gershman, S. J. \& Daw, N. D. Predictive

613 representations can link model-based reinforcement learning to model-free mechanisms. PLoS

$614 \quad$ Comput Biol 13, e1005768 (2017).

615 42. Koechlin, E. Prefrontal executive function and adaptive behavior in complex environments.

616 Current Opinion in Neurobiology 37, 1-6 (2016).

617 43. Szollosi, A. \& Newell, B. R. People as Intuitive Scientists: Reconsidering Statistical Explanations

618 of Decision Making. Trends in Cognitive Sciences 24, 1008-1018 (2020).

619 44. Hotaling, J. M., Jarvstad, A., Donkin, C. \& Newell, B. R. How to Change the Weight of Rare 620 Events in Decisions From Experience. Psychol Sci 30, 1767-1779 (2019).

621 45. Gershman, S. J., Horvitz, E. J. \& Tenenbaum, J. B. Computational rationality: A converging 622 paradigm for intelligence in brains, minds, and machines. Science 349, 273-278 (2015).

623 46. Hunter, L. E. \& Gershman, S. J. Reference-dependent preferences arise from structure learning.

624 http://biorxiv.org/lookup/doi/10.1101/252692 (2018) doi:10.1101/252692.

625 47. Lieder, F., Shenhav, A., Musslick, S. \& Griffiths, T. L. Rational metareasoning and the plasticity 626 of cognitive control. PLoS Comput Biol 14, e1006043 (2018).

627 48. Kleiner, M., Brainard, D. \& Pelli, D. What's new in Psychtoolbox-3? (2007).

628 49. Fox, C. R. \& Hadar, L. "Decisions from experience" = sampling error + prospect theory:

629 Reconsidering Hertwig, Barron, Weber \& Erev (2004). Judgment and Decision Making 1, 3 $630 \quad$ (2006).

631 